\title{
Report
}

\section{Large-Area Synthesis of High-Quality and Uniform Graphene Films on Copper Foils}

Xuesong Li, ${ }^{1}$ Weiwei Cai, ${ }^{1}$ Jinho An, ${ }^{1}$ Seyoung Kim, ${ }^{2}$ Junghyo Nah, ${ }^{2}$ Dongxing Yang, ${ }^{1}$ Richard Piner, ${ }^{1}$ Aruna Velamakanni, ${ }^{1}$ Inhwa Jung, ${ }^{1}$ Emanuel Tutuc, ${ }^{2}$ Sanjay K. Banerjee, ${ }^{2}$ Luigi Colombo, ${ }^{3}$ Rodney S. Ruoff $^{1 *}$

${ }^{1}$ Department of Mechanical Engineering and the Texas Materials Institute, 1 University Station C2200, The University of Texas at Austin, Austin, TX 78712-0292, USA. ${ }^{2}$ Department of Electrical and Computer Engineering, Microelectronics Research Center, The University of Texas at Austin, Austin, TX 78758, USA. ${ }^{3}$ Texas Instruments Incorporated, Dallas, TX 75243, USA.

*To whom correspondence should be addressed. E-mail: colombo@ti.com (L.C.); r.ruoff@mail.utexas.edu (R.S.R.)

Graphene has been attracting great interest because of its distinctive band structure and physical properties. Today, graphene is limited to small sizes since it is produced mostly by exfoliating graphite. We grew large area graphene films of the order of centimeters on copper substrates by chemical vapor deposition using methane. The films are predominantly single layer graphene with a small percentage, $<5 \%$, of the area having few layers, and are continuous across copper surface steps and grain boundaries. The low solubility of carbon in copper appears to help make this growth process self-limiting. Graphene film transfer processes to arbitrary substrates were also developed, and top gated field effect transistors fabricated on $\mathrm{Si} / \mathrm{SiO}_{2}$ substrates showed electron mobilities as high as $4300 \mathrm{~cm}^{2} \mathrm{~V}^{-1} \mathrm{~s}^{-1}$ at room temperature.

Graphene, a monolayer of $s p^{2}$-bonded carbon atoms, is a quasi-two-dimensional (2D) material. Graphene has been attracting great interest because of its distinctive band structure and physical properties (1). Today, the size of graphene films produced is limited to small sizes, usually less than $1000 \mu \mathrm{m}^{2}$, because it is produced mostly by exfoliating graphite, which is not a scalable technique. Graphene has also been synthesized by desorption of Si from $\mathrm{SiC}$ single crystal surfaces which yields a multilayered graphene structure that behaves like graphene $(2,3)$, and by a surface precipitation process of carbon in some transition metals (4-8).

Electronic application will require high-quality large area graphene that can be manipulated to make complex devices and integrated in silicon device flows. Field effect transistors (FETs), fabricated with exfoliated graphite have shown promising electrical properties $(9,10)$, but these devices will not meet the silicon device scaling requirements, principally power reduction and performance. One proposed device that could meet the silicon roadmap requirements beyond the 15$\mathrm{nm}$ node by S. K. Banerjee et al. (11) The device is a "BisFET" (bilayer pseudospin FET) device which is made up of two graphene layers separated by a thin dielectric. The ability to create this device can be facilitated by the availability of large area graphene. Making transparent electrode, another promising application of graphene, also requires large films $(6,12-14)$.

At this time, there is no pathway for the formation of a graphene layer that can be exfoliated from or transferred from the graphene synthesized on SiC but there is a way to grow and transfer graphene grown on metal substrates (5-7). Although graphene has been grown on a number of metals now, we still have the challenge of growing large area graphene. For example, graphene grown on Ni seems to be limited by its small grain size, presence of multilayers at the grain boundaries, and the high solubility of carbon $(6,7)$. We have developed a graphene chemical vapor deposition (CVD) growth process on copper foils (here, $25 \mu \mathrm{m}$ thick). The films grow directly on the surface by a surface catalyzed process and the film is predominantly graphene with less than $5 \%$ of the area having two- and three-layer graphene flakes. Under our processing conditions, the two- and three-layer flakes do not grow larger with time. One of the major benefits of our process is that it can be used to grow graphene on $300-\mathrm{mm}$ copper films on Si substrates (a standard process in $\mathrm{Si}$ technology). It is also well known that annealing of $\mathrm{Cu}$ can lead to very large grains.

As described in (15), we grew graphene on copper foils at temperatures up to $1000^{\circ} \mathrm{C}$ by CVD of carbon using a mixture of methane and hydrogen. Figure 1A shows a scanning electron microscope (SEM) image of graphene on a copper substrate where the $\mathrm{Cu}$ grains are clearly visible. A higher resolution image of graphene on $\mathrm{Cu}$ (Fig. 1B) shows the presence of Cu surface steps, graphene "wrinkles," and the presence of non-uniform dark flakes. The wrinkles associated with the thermal expansion coefficient difference between $\mathrm{Cu}$ and graphene are also found to cross $\mathrm{Cu}$ grain boundaries, indicating that the graphene film is continuous. The inset in Fig.1B shows transmission electron microscope (TEM) images of graphene and bilayer graphene. Using a process similar to that described in (16), the as-grown graphene can be easily transferred to alternative substrates such as $\mathrm{SiO}_{2} / \mathrm{Si}$ 
or glass (Fig. 1, C and D) for further evaluation and for various applications; a detailed transfer process is described in the supplemental section. The process and method used to transfer graphene from $\mathrm{Cu}$ was the same for the $\mathrm{SiO}_{2} / \mathrm{Si}$ substrate and the glass substrate. Although it is difficult to see the graphene on the $\mathrm{SiO}_{2} / \mathrm{Si}$ substrate, a similar graphene film from another $\mathrm{Cu}$ substrate transferred on glass clearly shows that it is optically uniform.

We used Raman spectroscopy to evaluate the quality and uniformity of graphene on $\mathrm{SiO}_{2} / \mathrm{Si}$ substrate. Figure 2 shows SEM and optical images with the corresponding Raman spectra and maps of the $\mathrm{D}, \mathrm{G}$, and $2 \mathrm{D}$ bands providing information on the defect density and film thickness. The Raman spectra are from the spots marked with the corresponding colored circles shown in the other panels (in Fig. 2, A and B, green arrows are used instead of circles so as to show the trilayer region more clearly). The thickness and uniformity of the graphene films were evaluated via color contrast under optical microscope (17) and Raman spectra (7, 18, 19). The Raman spectrum from the lightest pink background in Fig. 2B shows typical features of monolayer graphene; e.g., $~ 0.5$ G-to-2D intensity ratio, and a symmetric 2D band centered at $\sim 2680 \mathrm{~cm}^{-1}$ with a full width at half maximum (FWHM) of $\sim 33 \mathrm{~cm}^{-1}$. The second lightest pink "flakes" (blue circle) correspond to bilayer graphene and the darkest one (green arrow) is trilayer graphene. This thickness variation is more clearly shown in the SEM image in Fig. 2A. The D map, which has been associated with defects in graphene, in Fig. 2D is rather uniform and near the background level, except for regions where wrinkles are present and close to few-layer regions. The $\mathrm{G}$ and the 2D maps clearly show the presence of more than one layer in the flakes. In the wrinkled regions, there are peak height variations in both the G- and 2D-bands, and a broadening of the 2D band. An analysis of the intensity of the optical image over the whole sample $(1 \mathrm{~cm}$ by $1 \mathrm{~cm})$ showed that the area with the lightest pink color is more than $95 \%$, and all 40 Raman spectra randomly collected from such area shows monolayer graphene. There is only a small fraction of trilayer or few-layer $(<10)$ graphene, $<1 \%$, and the rest is bilayer graphene, $\sim 3$ to $4 \%$.

We grew films on $\mathrm{Cu}$ as a function of time and $\mathrm{Cu}$ foil thickness under isothermal and isobaric conditions. By using the process flow described in (15) we found that graphene growth on $\mathrm{Cu}$ is self-limited; growth that proceeded for more than 60 min yielded a similar structure to growth runs performed for about $10 \mathrm{~min}$. For times much less than 10 min, the Cu surface is usually not fully covered [SEM images of graphene on $\mathrm{Cu}$ with different growth time are shown in fig. S3 (15)]. The growth of graphene on $\mathrm{Cu}$ foils of varying thickness $(12.5,25$, and $50 \mu \mathrm{m})$ also yielded similar graphene structure with regions of double and triple flakes but neither discontinuous monolayer graphene for thinner $\mathrm{Cu}$ foils nor continuous multilayer graphene for thicker $\mathrm{Cu}$ foils, as we would have expected based on the precipitation mechanism. According to these observations, we concluded that graphene is growing by a surface-catalyzed process rather than a precipitation process as reported by others for $\mathrm{Ni}$ (5-7). Monolayer graphene formation caused by surface segregation or surface adsorption of carbon has also been observed on transition metals such as $\mathrm{Ni}$ and $\mathrm{Co}$ at elevated temperatures by Blakely and coauthors (20-22). However, when the metal substrates were cooled down to room temperature, thick graphite films were obtained because of precipitation of excess $C$ from these metals, in which the solubility of $C$ is relatively high.

In recent work, thin Ni films and a "fast cooling" process were used to suppress the amount of precipitated C. However, this process still yields films with a wide range of graphene layer thicknesses, from 1 to a few tens of layers, and with defects associated with fast cooling (5-7). Our results suggest that the graphene growth process is not one of $\mathrm{C}$ precipitation but rather a CVD process. The precise mechanism will require additional experiments to understand, but very low $\mathrm{C}$ solubility in $\mathrm{Cu}$ (23-25), and poor $\mathrm{C}$ saturation as a result of graphene surface coverage may be playing a role in limiting or preventing the precipitation process altogether at high temperature, similar to the case of impeding of carburization of $\mathrm{Ni}$ (26). This provides a pathway for growing self-limited graphene films.

In order to evaluate the electrical quality of the synthesized graphene, dual-gated field effect devices (FETs) using $\mathrm{Al}_{2} \mathrm{O}_{3}$ as the gate dielectric were fabricated and measured at room temperature. The data along with a device model which incorporates a finite density at the Dirac point, the dielectric as well as the quantum capacitances (9), are shown in Fig. 3. The extracted carrier mobility is $\sim 4300 \mathrm{~cm}^{2} \mathrm{~V}^{-1} \mathrm{~s}^{-1}$, with the residual carrier concentration at the Dirac point of $n_{0}=3.2 \times$ $10^{11} \mathrm{~cm}^{-2}$. These data suggest that the films are of reasonable quality, at least sufficient to continue improving the growth process to achieve a material quality equivalent to the exfoliated natural graphite.

\section{References and Notes}

1. A. K. Geim, K. S. Novoselov, Nat. Mater. 6, 183 (2007).

2. C. Berger et al., Science 312, 1991 (2006).

3. K. V. Emtsev et al., Nat. Mater. 8, 203 (2009).

4. P. W. Sutter, J.-I. Flege, E. A. Sutter, Nat. Mater. 7, 406 (2008).

5. Q. Yu et al., Appl. Phys. Lett. 93, 113103 (2008).

6. K. S. Kim et al., Nature 457, 706 (2009).

7. A. Reina et al., Nano Lett. 9, 30 (2009).

8. J. Coraux, A. T. N’Diaye, C. Busse, T. Michely, Nano Lett. 8, 565 (2008).

9. S. Kim et al., Appl. Phys. Lett. 94, 062107 (2009). 
10. M. C. Lemme et al., Solid-State Electron. 52, 514 (2008).

11. S. K. Banerjee, L. F. Register, E. Tutuc, D. Reddy, A. H. MacDonald, Electron Device Letters, IEEE 30, 158 (2009).

12. P. Blake et al., Nano Lett. 8, 1704 (2008).

13. R. R. Nair et al., Science 320, 1308 (2008).

14. X. Wang, L. Zhi, K. Müllen, Nano Lett. 8, 323 (2008).

15. See supporting material on Science Online.

16. A. Reina et al., J. Phys. Chem. C 112, 17741 (2008).

17. Z. H. Ni et al., Nano Lett. 7, 2758 (2007).

18. A. C. Ferrari et al., Phys. Rev. Lett. 97, 187401 (2006).

19. A. Das et al., Nat. Nanotechnol. 3, 210 (2008).

20. M. Eizenberg, J. M. Blakely, Surf. Sci. 82, 228 (1979).

21. M. Eizenberg, J. M. Blakely, J. Chem. Phys. 71, 3467 (1979).

22. J. C. Hamilton, J. M. Blakely, Surf. Sci. 91, 199 (1980).

23. R. B. McLellan, Scripta Metall. 3, 389 (1969).

24. G. Mathieu, S. Guiot, J. Carbané, Scripta Metall. 7, 421

(1973).

25. G. A. López, E. J. Mittemeijer, Scripta Materialia 51, 1 (2004).

26. R. Kikowatz, K. Flad, G. Horz, J. Vac. Sci. Technol. A 5, (1987).

27. We would like to thank the Nanoelectronic Research Initiative (NRI-SWAN; \#2006-NE-1464), the DARPA CERA Center, and The University of Texas at Austin for support.

\section{Supporting Online Material}

\section{www.sciencemag.org/cgi/content/full/1171245/DC1}

Materials and Methods

Figs. S1 to S3

22 January 2009; accepted 9 April 2009

Published online 7 May 2009; 10.1126/science.1171245

Include this information when citing this paper.

Fig. 1. (A) An SEM image of graphene on a copper foil with a growth time of $30 \mathrm{~min}$ and (B) a high-resolution SEM image showing a $\mathrm{Cu}$ grain boundary and steps, 2- and 3-layer graphene flakes, and graphene wrinkles. Inset in (B) shows TEM images of folded graphene edges. ( $C$ and $\mathbf{D}$ ) Graphene films transferred onto a $\mathrm{SiO}_{2} / \mathrm{Si}$ substrate and a glass plate, respectively.

Fig. 2. (A) $\mathrm{SEM}$ image of graphene transferred on $\mathrm{SiO}_{2} / \mathrm{Si}$ (285-nm-thick oxide layer) showing wrinkles, and 2- and 3layer regions. (B) Optical microscope image of the same regions as (A). (C) Raman spectra from the marked spots with corresponding colored circles or arrows showing the presence of graphene, 2 layers of graphene and 3 layers of graphene; (D to F) Raman maps of the D (1300 to $1400 \mathrm{~cm}^{-}$ $\left.{ }^{1}\right)$, G (1560 to $\left.1620 \mathrm{~cm}^{-1}\right)$, and 2D (2660 to $\left.2700 \mathrm{~cm}^{-1}\right)$ bands, respectively (WITec alpha300, $\lambda_{\text {laser }}=532 \mathrm{~nm}, \sim 500-\mathrm{nm}$ spot size, $100 \times$ objector). Scale bars are $5 \mu \mathrm{m}$.

Fig. 3. (A) Optical microscope image of a graphene FET. (B) Device resistance vs top-gate voltage $\left(V_{\mathrm{TG}}\right)$ with different back-gate $\left(V_{\mathrm{BG}}\right)$ biases and versus $V_{\mathrm{TG}}-V_{\text {Dirac,TG }}\left(V_{\mathrm{TG}}\right.$ at the Dirac point), with a model fit (solid line). 


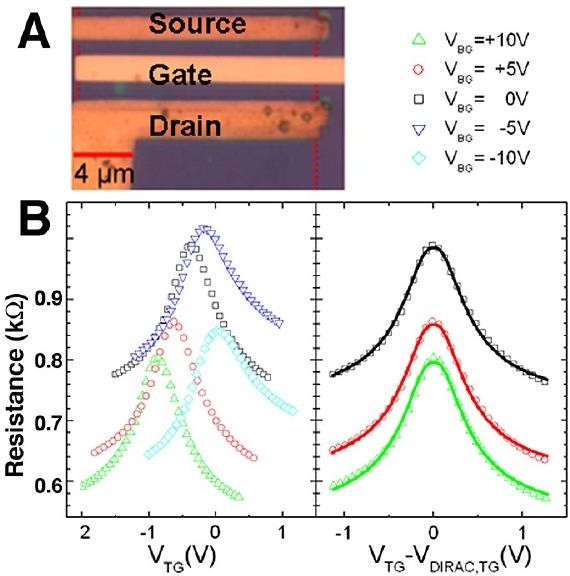

